\title{
Positive Solutions for a Class of Quasilinear Schrödinger Equations with Nonlocal Term
}

\author{
Peng Liao, Rui Ping, Shaoxiong Chen* \\ School of Mathematics, Yunnan Normal University, Kunming, China \\ Email: *gxmail@126.com
}

How to cite this paper: Liao, P., Ping, R. and Chen, S.X. (2022) Positive Solutions for a Class of Quasilinear Schrödinger Equations with Nonlocal Term. Journal of Applied Mathematics and Physics, 10, 347-359. https://doi.org/10.4236/jamp.2022.102027

Received: January 17, 2022

Accepted: February 15, 2022

Published: February 18, 2022

Copyright $\odot 2022$ by author(s) and Scientific Research Publishing Inc. This work is licensed under the Creative Commons Attribution International License (CC BY 4.0).

http://creativecommons.org/licenses/by/4.0/

\begin{abstract}
This paper is considered the existence of positive solutions for a class of generalized quasilinear Schrödinger equations with nonlocal term in $\mathbb{R}^{N}$ which have appeared from plasma physics, as well as high-power ultrashort laser in matter. We use a charge of variables and obtain the existence of solutions via minimization argument.
\end{abstract}

\section{Keywords}

Quasilinear Schrödinger Equation, Minimization, Implicit Function Theorem

\section{Introduction}

In this paper, we consider investigating the existence of solutions for the following generalized quasilinear Schrödinger equation with nonlocal term

$$
\begin{aligned}
& -\operatorname{div}\left(g^{2}(u) \nabla u\right)+g(u) g^{\prime}(u)|\nabla u|^{2}+V(x) u \\
& =\lambda\left[|x|^{-\mu} *|u|^{p}\right]|u|^{p-2} u+\beta|u|^{q-1} u,
\end{aligned}
$$

where $N \geq 3,0<\mu<N, \quad \beta<\frac{N-2}{2 N}, 1 \leq q \leq \frac{N}{N-2}, \frac{2 N-\mu}{N} \leq p<\frac{2 N-\mu}{N-2}$, the function $V \in C\left(\mathbb{R}^{N}, \mathbb{R}^{+}\right), g$ is a $\mathcal{C}^{1}$ function with $g^{\prime}(t) \leq 0$ for all $t>0$, $g(0)=0, \lim _{t \rightarrow+\infty} g(t)=a$.

When $g(u)=1$, (1.1) boils down to the so called nonlinear Choquard or Choquard-Pekar equation

$$
-\Delta u+V(x) u=\lambda\left[|x|^{-\mu} *|u|^{p}\right]|u|^{p-2} u+\beta|u|^{q-1} u
$$

Such like equation has several physical origins. The problem

$$
-\Delta u+u=\left[|x|^{-1} *|u|^{2}\right] u,
$$


appeared at least as early as in 1954, in a work by Pekar describing the quantum mechanics of a polaron at rest [1]. In 1976, Choquard used (1.3) to describe an electron trapped in its own hole and in a certain approximation to Hartree-Fock theory of one component plasma [2]. In 1996, Penrose proposed (1.3) as a model of self-gravitating matter, in a program in which quantum state reduction is understood as a gravitational phenomenon [3]. In this context, equation of type (1.3) is usually called the nonlinear Schrödinger-Newtonequation. The first investigations for the existence and symmetry of the solutions to (1.3) go back to the works of Lieb [2] and Lions [4]. In [2], by using symmetric decreasing rearrangement inequalities, Lieb proved that the ground state solution of Equation (1.3) is radial and unique up to translations. Lions [4] showed the existence of a sequence of radially symmetric solutions. Ma and Zhao [5] considered the generalized Choquard equation

$$
-\Delta u+u=\left[|x|^{-\mu} *|u|^{q}\right]|u|^{q-2} u(q \geq 2),
$$

and proved that every positive solution of it is radially symmetric and monotone decreasing about some fixed point, under the assumption that a certain set of real numbers, defined in terms of $N$, and $q$, is nonempty. Under the same assumption, Cingolani, Clapp, and Secchi [6] gave some existence and multiplicity results in the electromagnetic case and established the regularity and some decay asymptotically at infinity of the ground states. In [7], Moroz and Van Schaftingen eliminated this restriction and showed the regularity, positivity and radial symmetry of the ground states for the optimal range of parameters and derived decay asymptotically at infinity for them as well. Moreover, they [8] also obtained a similar conclusion under the assumption of Berestycki-Lions type nonlinearity. We point out that the existence, multiplicity, and concentration of such like equation have been established by many authors. We refer the readers to [9] [10] for the existence of sign-changing solutions, [11] [12] for the existence and concentration behavior of the semiclassical solutions and [13] for the critical nonlocal part with respect to the Hardy-Littlewood-Sobolev inequality. For more details associated with the Choquard equation, please refer to [14] [15] [16] and the references in. Li, Teng, Zhang, Nie [17] investigate the existence of solutions for the following generalized quasilinear Schrödinger equation with nonlocal term

$$
\begin{aligned}
& -\operatorname{div}\left(g^{2}(u) \nabla u\right)+g(u) g^{\prime}(u)+|\nabla u|^{2}+V(x) u \\
& =\lambda\left[|x|^{-\mu} *|u|^{p}\right]|u|^{p-2} u, x \in \mathbb{R}^{N}
\end{aligned}
$$

and prove the existence of solution.

In this paper, our main ideas come from [18] and the assumption of $g$ from [19]. Our purpose is to search for the existence of nontrivial solutions of (1.1) by implicit function theorem. For convenience, we introduce several notations: $C$ denotes a positive (possibly different) constant, $L^{p}\left(\mathbb{R}^{N}\right)$ denotes the usual Lebesgue space with norms $\|u\|_{L^{p}\left(\mathbb{R}^{N}\right)}=\left(\int_{\mathbb{R}^{N}}|u|^{p} \mathrm{~d} x\right)^{\frac{1}{p}}, 1 \leq p<\infty, C_{0}^{\infty}\left(\mathbb{R}^{N}\right)$ be the collec- 
tion of smooth functions with compact support. Next, we introduce the energy functional of Equation (1.1)

$$
\begin{aligned}
I(u)= & \frac{1}{2} \int_{\mathbb{R}^{N}}\left[g^{2}(u)|\nabla u|^{2}+V(x) u^{2}\right] \mathrm{d} x \\
& -\frac{\lambda}{2 p} \int_{\mathbb{R}^{N}}\left[|x|^{-\mu} *|u|^{p}\right]|u|^{p} \mathrm{~d} x-\frac{\beta}{q+1} \int_{\mathbb{R}^{N}}|u|^{q+1} \mathrm{~d} x
\end{aligned}
$$

however, Jis not well defined in $H^{1}\left(\mathbb{R}^{N}\right)$ because of the term $\int_{\mathbb{R}^{N}} g^{2}(u)|\nabla u|^{2} \mathrm{~d} x$. To overcome this difficulty, we make a change of variable constructed by Shen and Wang in [20]: $v=G(u)=\int_{0}^{u} g(s) \mathrm{d} s$, then,

$$
\begin{aligned}
J(v)= & \frac{1}{2} \int_{\mathbb{R}^{N}}\left[|\nabla v|^{2}+V(x)\left|G^{-1}(v)\right|^{2}\right] \mathrm{d} x \\
& -\frac{\lambda}{2 p} \int_{\mathbb{R}^{N}}\left(\left[|x|^{-\mu} *\left|G^{-1}(v)\right|^{p}\right]\left|G^{-1}(v)\right|^{p}\right) \mathrm{d} x+\int_{\mathbb{R}^{N}}\left[\frac{\beta}{q+1}\left|G^{-1}(v)\right|^{q+1}\right] \mathrm{d} x \\
= & \frac{1}{2} \int_{\mathbb{R}^{N}}\left[|\nabla v|^{2}+V(x)\left|G^{-1}(v)\right|^{2}\right] \mathrm{d} x \\
& -\frac{\lambda}{2 p} \int_{\mathbb{R}^{2 N}}\left(\frac{\left|G^{-1}(v(y))\right|^{p}\left|G^{-1}(v(x))\right|^{p}}{|x-y|^{\mu}}\right) \mathrm{d} x \mathrm{~d} y-\int_{\mathbb{R}^{N}}\left[\frac{\beta}{q+1}\left|G^{-1}(v)\right|^{q+1}\right] \mathrm{d} x
\end{aligned}
$$

We say that $u$ is a weak solution of (1.1), if

$$
\begin{aligned}
\left\langle I^{\prime}(u), \varphi\right\rangle= & \int_{R^{N}}\left(g^{2}(u) \nabla u \nabla \varphi+g(u) g^{\prime}(u)|\nabla u|^{2} \varphi+V(x) u \varphi\right) \mathrm{d} x \\
& -\lambda \int_{\mathbb{R}^{N}}\left[|x|^{-\mu} *|u|^{p}\right]|u|^{p-2} u \varphi-\beta \int_{\mathbb{R}^{N}}|u|^{q-1} u \varphi \mathrm{d} x
\end{aligned}
$$

for all $\varphi \in C_{0}^{\infty}\left(\mathbb{R}^{N}\right)$. Let $\varphi=\left(\frac{1}{g(u)} \psi\right)$, by [20], we know that the above formula is equivalent to

$$
\begin{aligned}
\left\langle J^{\prime}(v), \psi\right\rangle= & \int_{\mathbb{R}^{N}} \nabla v \nabla \psi+V(x) \frac{G^{-1}(v)}{g\left(G^{-1}(v)\right)} \psi \mathrm{d} x \\
& -\lambda \int_{\mathbb{R}^{N}} \frac{\left[|x|^{-2} *\left|G^{-1}(v)\right|^{p}\right]\left|G^{-1}(v)\right|^{p-2} G^{-1}(v) \psi}{g\left(G^{-1}(v)\right)} \mathrm{d} x \\
& -\beta \int_{\mathbb{R}^{N}} \frac{\left|G^{-1}(v)\right|^{q-1} G^{-1}(v)}{g\left(G^{-1}(v)\right)} \psi \mathrm{d} x
\end{aligned}
$$

for all $\psi \in C_{0}^{\infty}\left(\mathbb{R}^{N}\right)$. Therefore, in order to find the solution of (1.1), it suffices to study the solution of following equation:

$$
\begin{aligned}
& -\Delta v+V(x) \frac{G^{-1}(v)}{g\left(G^{-1}(v)\right)}-\lambda \frac{\left[|x|^{-\mu} *\left|G^{-1}(v)\right|^{p}\right]\left|G^{-1}(v)\right|^{p-2} G^{-1}(v)}{g\left(G^{-1}(v)\right)} \\
& -\beta \frac{\left|G^{-1}(v)\right|^{q-1} G^{-1}(v)}{g\left(G^{-1}(v)\right)}=0
\end{aligned}
$$

$J$ is defined on the space 


$$
H_{V}^{1}\left(\mathbb{R}^{N}\right)=\left\{v \in H^{1}\left(\mathbb{R}^{N}\right): \int_{\mathbb{R}^{N}}|\nabla v|^{2}+V(x) v^{2} \mathrm{~d} x<+\infty\right\}
$$

we can define the norm on $H_{V}^{1}\left(\mathbb{R}^{N}\right)$ by

$$
\|v\|_{H_{V}^{1}\left(\mathbb{R}^{N}\right)}^{2}=\int_{\mathbb{R}^{N}}|\nabla v|^{2}+V(x) v^{2} \mathrm{~d} x
$$

then, $H_{V}^{1}\left(\mathbb{R}^{N}\right)$ is a Banach space. In the following, we always assume $V \in C\left(\mathbb{R}^{N}, \mathbb{R}^{+}\right)$and $\inf _{\mathbb{R}^{N}} V(x) \geq 1$. Let us consider the following assumptions of potential function $V(x)$ :

$\left(V_{1}\right) \lim _{|x| \rightarrow \infty} V(x)=+\infty$;

$\left(V_{2}\right) V(x)$ is radially symmetric.

Next, we will introduce the properties of some functions.

Lemma 1.1. [19] The function $g(t), G^{-1}(t), G(t)$ enjoys the following properties.

$\left(g_{1}\right)$ the function $G(t)$ and $G^{-1}(t)$ are strictly increasing and odd;

$\left(g_{2}\right)|t| \leq\left|G^{-1}(t)\right| \leq|t| / a$ for all $t \in \mathbb{R}$;

$\left(g_{3}\right) G^{-1}(t) / t$ is nondecreasing for all $t \in \mathbb{R}$ and $\lim _{t \rightarrow 0} G^{-1}(t) / t=1$, $\lim _{t \rightarrow \infty} G^{-1}(t) / t=1 / a$;

$\left(g_{4}\right) \quad t^{2} \leq(t / g(t)) G(t) \leq t^{2} / a$ for all $t \in \mathbb{R}$.

Next, we set forth some preliminary results.

\section{Preliminary Results}

To begin with, we prove some functions are continuous, more detailed see [21].

Lemma 2.1 If $\left\|v_{n}-v\right\|_{H_{V}^{1}\left(\mathbb{R}^{N}\right)} \rightarrow 0$, then $\int_{\mathbb{R}^{N}} V(x) G^{-1}\left(v_{n}\right)^{2}-V(x) G^{-1}(v)^{2} \mathrm{~d} x \rightarrow 0$.

Proof: By sobolev imbedding inequality, Lemma1.1 and definition of $g$, we have

$$
\begin{aligned}
& \int_{\mathbb{R}^{N}} V(x) G^{-1}\left(v_{n}\right)^{2}-V(x) G^{-1}(v)^{2} \mathrm{~d} x \\
& =2 \int_{\mathbb{R}^{N}} V(x) \frac{G^{-1}\left(v+\theta\left(v_{n}-v\right)\right)}{g\left(G^{-1}\left(v+\theta\left(v_{n}-v\right)\right)\right)}\left(v_{n}-v\right) \mathrm{d} x \\
& \leq \frac{2}{a^{2}} \int_{\mathbb{R}^{N}} V(x)\left|v+\theta\left(v_{n}-v\right)\right|\left|v_{n}-v\right| \mathrm{d} x \\
& \leq \frac{2}{a^{2}} \int_{\mathbb{R}^{N}} V(x)\left|v_{n}-v\right|^{2} \mathrm{~d} x \int_{R^{N}} V(x)\left|v+\theta\left(v_{n}-v\right)\right|^{2} \mathrm{~d} x \\
& \leq \frac{2}{a^{2}} \int_{\mathbb{R}^{N}} V(x)\left|v_{n}-v\right|^{2} \mathrm{~d} x\left(\int_{\mathbb{R}^{N}} V(x) v^{2} \mathrm{~d} x+\int_{\mathbb{R}^{N}} V(x)\left|v_{n}-v\right|^{2} \mathrm{~d} x\right) \\
& \leq \frac{2}{a^{2}}\left\|v_{n}-v\right\|_{H_{V}^{1}\left(\mathbb{R}^{N}\right)}\left(C+\left\|v_{n}-v\right\|_{H_{V}^{1}\left(\mathbb{R}^{N}\right)}\right) \rightarrow 0, n \rightarrow+\infty
\end{aligned}
$$

where $\theta \in(0,1)$.

Lemma 2.2. The map: $v \rightarrow G^{-1}(v)$ from $H_{V}^{1}\left(\mathbb{R}^{N}\right)$ into $L^{r}\left(\mathbb{R}^{N}\right)$ is continuous for $2 \leq r<2^{*}$.

Proof: By the definition of $g$, we have 


$$
\begin{aligned}
\int_{\mathbb{R}^{N}}\left|G^{-1}\left(v_{n}\right)-G^{-1}(v)\right|^{r} \mathrm{~d} x & \leq \int_{\mathbb{R}^{N}}\left|G^{-1}\left(v_{n}\right)-G^{-1}(v)\right|^{r} \mathrm{~d} x \\
& \leq \frac{1}{a^{r}} \int_{\mathbb{R}^{N}}\left(\left|v_{n}\right|^{r}+|v|^{r}\right) \mathrm{d} x .
\end{aligned}
$$

Assume $v_{n} \rightarrow v$ in $H_{V}^{1}\left(\mathbb{R}^{N}\right)$, moreover, the imbedding from $H_{V}^{1}\left(\mathbb{R}^{N}\right)$ into $L^{r}\left(\mathbb{R}^{N}\right)$ is compact where $r \in\left[2,2^{*}\right)$, from Lemma 3.4 [22], we get the result.

Next, we introduce some minimization with corresponding energy functional and define

$$
m_{b}=\inf _{u \in M_{b}} E(u)
$$

where

$$
M_{b}=\left\{u \in H^{1}\left(\mathbb{R}^{N}\right):\|u\|_{L^{p+1}}=b\right\}, a>0
$$

and

$$
E(u)=\frac{1}{2} \int_{\mathbb{R}^{N}}\left[g^{2}(u)|\nabla u|^{2}+V(x) u^{2}\right] d x-\frac{\beta}{q+1} \int_{\mathbb{R}^{N}}|u|^{q+1} \mathrm{~d} x .
$$

We also define

$$
\omega_{b}=\inf _{v \in W_{b}} F(v)
$$

where

$$
W_{b}=\left\{v \in H_{V}^{1}\left(\mathbb{R}^{N}\right):\left\|G^{-1}(v)\right\|_{L^{p+1}}=b\right\}, b>0
$$

and

$$
F(v)=\frac{1}{2} \int_{\mathbb{R}^{N}}\left(|\nabla v|^{2}+V(x) G^{-1}(v)^{2}\right) \mathrm{d} x-\frac{\beta}{q+1} \int_{\mathbb{R}^{N}}\left|G^{-1}(v)\right|^{q+1} \mathrm{~d} x
$$

Therefore, we have following fact.

Lemma 2.3. $m_{b}=\omega_{b}$ for every $a>0$.

Proof: For any $v \in W_{b}$, let $u=G^{-1}(v)$, from the definition of $g$, we get

$$
\begin{aligned}
& \int_{\mathbb{R}^{N}}|\nabla u|^{2} \mathrm{~d} x=\int_{\mathbb{R}^{N}} \frac{|\nabla v|^{2}}{g^{2}\left(G^{-1}(v)\right)} \mathrm{d} x \leq \frac{1}{a^{2}} \int_{\mathbb{R}^{N}}|\nabla v|^{2} \mathrm{~d} x<+\infty, \\
& \int_{R^{N}} u^{2} \mathrm{~d} x \leq \int_{\mathbb{R}^{N}} V(x) G^{-1}(v)^{2} \mathrm{~d} x<+\infty,
\end{aligned}
$$

so $u \in M_{b}$. It follows that $F(v)=E\left(G^{-1}(v)\right)=E(u) \geq m_{b}$, hence $\omega_{b} \geq m_{b}$, moreover, for any $u \in M_{b}$, let $v=G(u)$, then $u=G^{-1}(v)$. We assume $E(u)<+\infty$, since $u \in H_{V}^{1}\left(\mathbb{R}^{N}\right), \quad 2<q+1<2^{*}$, then $u \in L^{q+1}\left(\mathbb{R}^{N}\right)$. We have

$$
\frac{1}{2} \int_{\mathbb{R}^{N}}\left[g^{2}(u)|\nabla u|^{2}+V(x) u^{2}\right] \mathrm{d} x=E(u)+\frac{\beta}{q+1} \int_{\mathbb{R}^{N}}|u|^{q+1} \mathrm{~d} x<+\infty .
$$

Then $\int_{\mathbb{R}^{N}} V(x) G^{-1}(v)^{2}=\int_{\mathbb{R}^{N}} V(x) u^{2} \mathrm{~d} x<+\infty$. It shows that $v \in W_{a}$, which implies that $E(u)=E\left(G^{-1}(v)\right)=F(v) \geq \omega_{b}$, hence $m_{b} \geq \omega_{b}$, this completes the proof.

Lemma 2.4. 1) $F(v)$ is well defined and continuous for $2 \leq r \leq 2^{*}$.

2) $F(v)$ is Gateaux-differentiable. For $v \in H_{V}^{1}\left(\mathbb{R}^{N}\right)$, the $G$-derivative $F^{\prime}(v)$ is a continuous function, and $F^{\prime}(v)$ is continuous in $v$ in the strongly-weak 
topology, that is, if $v_{n} \rightarrow v$ strongly in $H_{V}^{1}\left(\mathbb{R}^{N}\right)$, then $F^{\prime}\left(v_{n}\right) \rightarrow F^{\prime}(v)$ weakly.

Proof: (1) For any $v \in H_{V}^{1}\left(\mathbb{R}^{N}\right)$, we have $\int_{\mathbb{R}^{N}}\left|G^{-1}(v)\right|^{q+1} \mathrm{~d} x \leq C \int_{\mathbb{R}^{N}}|v|^{q+1} \mathrm{~d} x<+\infty$, then

$$
\begin{aligned}
F(v) & =\frac{1}{2} \int_{\mathbb{R}^{N}}|\nabla v|^{2}+V(x) G^{-1}(v)^{2} \mathrm{~d} x-\frac{\beta}{q+1} \int_{\mathbb{R}^{N}}\left|G^{-1}(v)\right|^{q+1} \mathrm{~d} x \\
& \leq \frac{1}{2 a^{2}} \int_{\mathbb{R}^{N}}|\nabla v|^{2}+V(x) v^{2} \mathrm{~d} x+\left|\frac{\beta}{q+1}\right| \int_{\mathbb{R}^{N}}\left|G^{-1}(v)\right|^{q+1} \mathrm{~d} x \\
& \leq \frac{1}{2 a^{2}} \int_{\mathbb{R}^{N}}|\nabla v|^{2}+V(x) v^{2} \mathrm{~d} x+\frac{\beta}{(q+1) a^{q+1}} \int_{\mathbb{R}^{N}}|v|^{q+1} \mathrm{~d} x<+\infty .
\end{aligned}
$$

with the proof of continuity, note that $J$ consists of three terms. By Lemma 1.1, we need to check the superlinear term only.

$$
\begin{aligned}
& \left.\left|\frac{1}{q+1} \int_{\mathbb{R}^{N}}\right| G^{-1}\left(v_{n}\right)\right|^{q+1} \mathrm{~d} x-\frac{1}{q+1} \int_{\mathbb{R}^{N}}\left|G^{-1}(v)\right|^{q+1} \mathrm{~d} x \mid \\
& =\left|\int_{0}^{1} \mathrm{~d} t \int_{\mathbb{R}^{N}} \frac{\left|G^{-1}\left(v+t\left(v_{n}-v\right)\right)\right|^{q-1} G^{-1}\left(v+t\left(v_{n}-v\right)\right)}{g\left(G^{-1}\left(v+t\left(v_{n}-v\right)\right)\right)}\left(v_{n}-v\right) \mathrm{d} x\right| \\
& \leq C \int_{0}^{1} \int_{\mathbb{R}^{N}}\left|v+t\left(v_{n}-v\right)\right|^{q}\left|v_{n}-v\right| \mathrm{d} t \mathrm{~d} x \\
& \leq\left\|v+\left.t\left(v_{n}-v\right)\right|^{q}\right\|_{L^{2}\left(\mathbb{R}^{N}\right)}\left\|v_{n}-v\right\|_{L^{2}\left(\mathbb{R}^{N}\right)} \\
& \leq C\left\|v_{n}-v\right\|_{H_{V}^{1}}
\end{aligned}
$$

where $1 \leq q \leq \frac{N}{N-2}$.

For (2) we consider the second and the third terms of the functional $J$, we see for $\phi \in H_{V}^{1}\left(\mathbb{R}^{N}\right)$, using Hölder inequality, we get

$$
\begin{aligned}
& \left|\frac{1}{2 t} \int_{\mathbb{R}^{N}} V(x)\left(G^{-1}(v+t \phi)^{2}-G^{-1}(v)^{2}\right) \mathrm{d} x-\int_{\mathbb{R}^{N}} \frac{V(x) G^{-1}(v)}{g\left(G^{-1}(v)\right)} \phi \mathrm{d} x\right| \\
& =\mid \int_{0}^{1} \mathrm{~d} s \int_{\mathbb{R}^{N}} V(x)\left(\frac{G^{-1}(v+t s \phi)}{g\left(G^{-1}(v+t s \phi)\right)}-\frac{G^{-1}(v)}{g\left(G^{-1}(v)\right)}|\phi \mathrm{d} x|\right. \\
& \leq \int_{0}^{1} \mathrm{~d} s\left(\left.\int_{\mathbb{R}^{N}} V(x)\left|\frac{G^{-1}(v+t s \phi)}{g\left(G^{-1}(v+t s \phi)\right)}-\frac{G^{-1}(v)}{g\left(G^{-1}(v)\right)}\right|^{2} \mathrm{~d} x\right|^{\frac{1}{2}} \int_{0}^{1} \mathrm{~d} s\left(\int_{\mathbb{R}^{N}} V(x) \phi^{2} \mathrm{~d} x\right)^{\frac{1}{2}}\right.
\end{aligned}
$$

Using the definition of $g$ and Lemma 1.1, we know

$$
\begin{aligned}
\left|\frac{G^{-1}(v+t s \phi)}{g\left(G^{-1}(v+t s \phi)\right)}-\frac{G^{-1}(v)}{g\left(G^{-1}(v)\right)}\right|^{2} & \leq\left|G^{-1}(v+t s \phi)+G^{-1}(v)\right|^{2} \\
& \leq C\left(\left|G^{-1}(v+t s \phi)\right|^{2}+\left|G^{-1}(v)\right|^{2}\right) \\
& \leq C\left(|v+t s \phi|^{2}+|v|^{2}\right) \\
& \leq C\left(|v|^{2}+|\phi|^{2}\right) .
\end{aligned}
$$


By the dominated convergence theorem

$$
\left|\int_{0}^{1} \mathrm{~d} s \int_{\mathbb{R}^{N}} V(x)\left(\frac{G^{-1}(v+t s \phi)}{g\left(G^{-1}(v+t s \phi)\right)}-\frac{G^{-1}(v)}{g\left(G^{-1}(v)\right)}\right) \phi \mathrm{d} x\right| \rightarrow 0 \quad(t \rightarrow 0)
$$

For the third term, we have

$$
\begin{aligned}
& \left|\frac{1}{t} \int_{\mathbb{R}^{N}} \frac{1}{q+1}\left(\left|G^{-1}(v+t \phi)\right|^{q+1}-\left|G^{-1}(v)\right|^{q+1}\right) \mathrm{d} x-\int_{\mathbb{R}^{N}} \frac{G^{-1}(v)^{q-1} G^{-1}(v)}{g\left(G^{-1}(v)\right)} \phi \mathrm{d} x\right| \\
& =\left|\frac{1}{t} \int_{0}^{1} \mathrm{~d} s \int_{\mathbb{R}^{N}}\left(\frac{\left|G^{-1}(v+t s \phi)\right|^{q-1} G^{-1}(v+t s \phi)}{g\left(G^{-1}(v+t s \phi)\right)}-\frac{\left|G^{-1}(v)\right|^{q-1} G^{-1}(v)}{g\left(G^{-1}(v)\right)}\right) \phi \mathrm{d} x\right|
\end{aligned}
$$

Similarly to above, by the dominated convergence theorem

$$
\lim _{t \rightarrow 0}\left|\frac{1}{t} \int_{0}^{1} \mathrm{~d} s \int_{\mathbb{R}^{N}}\left(\frac{\left|G^{-1}\right|^{q-1}(v+t s \phi)}{g\left(G^{-1}(v+t s \phi)\right)}-\frac{\left|G^{-1}\right|^{q-1}(v) G^{-1}(v)}{g\left(G^{-1}(v)\right)}\right) \phi \mathrm{d} x\right|=0
$$

The Gateaux derivative $J^{\prime}(v)$ has the form

$$
\begin{aligned}
\left\langle J^{\prime}(v), \phi\right\rangle & =\int_{\mathbb{R}^{N}} \nabla v \nabla \phi \mathrm{d} x+\int_{\mathbb{R}^{N}} \frac{V(x) G^{-1}(v)}{g\left(G^{-1}(v)\right)} \phi \mathrm{d} x-\int_{\mathbb{R}^{N}} \frac{\left|G^{-1}(v)\right|^{q-1} G^{-1}(v)}{g\left(G^{-1}(v)\right)} \phi \mathrm{d} x \\
& \leq C\|v\|_{H_{V}^{1}\left(\mathbb{R}^{N}\right)}\|\phi\|_{H_{V}^{1}\left(\mathbb{R}^{N}\right)}+C\left\|G^{-1}(v)\right\|_{L^{2 q}\left(\mathbb{R}^{N}\right)}\|\phi\|_{L^{2}\left(\mathbb{R}^{N}\right)}
\end{aligned}
$$

from Sobolev imbedding theorem, we get $J^{\prime}(v)$ is a continuous linear functional on $H_{V}^{1}\left(\mathbb{R}^{N}\right)$.

Finally, the continuity with strong-weak topology is easy to check, as $v_{n} \rightarrow v$ in $H_{V}^{1}\left(\mathbb{R}^{N}\right)$, for any $\phi \in H_{V}^{1}\left(\mathbb{R}^{N}\right)$,

$$
\int_{\mathbb{R}^{N}} V(x) \frac{G^{-1}\left(v_{n}\right)}{g\left(G^{-1}\left(v_{n}\right)\right)}-V(x) \frac{G^{-1}(v)}{g\left(G^{-1}(v)\right)} \mathrm{d} x \rightarrow 0 .
$$

Remark 2.5. Lemma 2.4 does not show that $F(v)$ is $C^{\infty}$, so we cannot use the Lagrange multiplier theorem. But we can get our conclusion we want exactly by a similar argument for the Lagrange multiplier theorem. Next, we state our main conclusion. The idea of our proof is based on the work in [18] [22] [23].

\section{Main Conclusion}

Theorem 3.1. Let $N \geq 3,1 \leq q<\frac{N}{N-2}, \frac{2 N-\mu}{N} \leq p<\frac{2 N-\mu}{N-2}, \beta \in \mathbb{R}$ and $q<p$. Assume $\left(V_{1}\right)$ or $\left(V_{2}\right)$ holds. Then for every $b>0$, there exists $\lambda(b) \in \mathbb{R}$ such that Equation (1.1) with $\lambda=\lambda(b)$ has a positive weak solution $u \in M_{b}$.

Remark 3.2. From the assumption of $V$, we know $H_{V}^{1}\left(\mathbb{R}^{N}\right)$ embedding into $L^{p}\left(\mathbb{R}^{N}\right)$ is compact. In the process of the proof of theorem 3.1, it is important for us to construct auxiliary function, then by implicit function theorem to prove it and lemma 3.4 [22] play a great role in this paper. Moreover, when $\frac{N-2}{N}<q \leq 2^{*}$ 
is a open question for Equation (1.1), someone could do it if they are interested.

Proof of Theorem 3.1: Step 1: By the assumptions of $\left(V_{1}\right)$ or $\left(V_{2}\right), \omega_{b}$ is achieved at some $0 \leq v_{b} \leq W_{b}$ with $v_{b} \neq 0$. Let $\left\{v_{n}\right\} \in W_{b}$ be a minimizing sequence for $\omega_{b}$. Set $u_{n}=G^{-1}\left(v_{n}\right)$. Then $\left\{u_{n}\right\} \in M_{b}$ is a minimizing sequence for $m_{b}$. We can assume $u_{n} \geq 0$. It shows that $E\left(u_{n}\right) \rightarrow m_{b}$, so there exists $C>0$ such that

$$
\begin{aligned}
C & \geq E\left(u_{n}\right) \\
& =\frac{1}{2} \int_{\mathbb{R}^{N}}\left[g^{2}\left(u_{n}\right)\left|\nabla u_{n}\right|^{2}+V(x) u_{n}^{2}\right] \mathrm{d} x-\frac{\beta}{q+1} \int_{R^{N}}\left|u_{n}\right|^{q+1} \mathrm{~d} x \\
& \geq \frac{1}{2} \int_{\mathbb{R}^{N}}\left[|\nabla u|^{2}+\left|u_{n}\right|^{2}\right] \mathrm{d} x-\frac{\beta}{q+1} \int_{\mathbb{R}^{N}}\left|u_{n}\right|^{q+1} \mathrm{~d} x .
\end{aligned}
$$

By Hölder inequality,

$$
\begin{aligned}
\int_{\mathbb{R}^{N}}\left|u_{n}\right|^{q+1} \mathrm{~d} x & \leq\left(\int_{\mathbb{R}^{N}}\left|u_{n}\right|^{2} \mathrm{~d} x\right)^{\frac{\lambda(q+1)}{2}}\left(\int_{\mathbb{R}^{N}}\left|u_{n}\right|^{p+1} \mathrm{~d} x\right)^{\frac{(1-\lambda)(q+1)}{p+1}} \\
& \leq \frac{\lambda(q+1)}{2} \int_{\mathbb{R}^{N}}\left|u_{n}\right|^{2} \mathrm{~d} x+\frac{(1-\lambda)(q+1)}{p+1} \int_{\mathbb{R}^{N}}\left|u_{n}\right|^{p+1} \mathrm{~d} x \\
& =\frac{\lambda(q+1)}{2} \int_{\mathbb{R}^{N}}\left|u_{n}\right|^{2} \mathrm{~d} x+\frac{(1-\lambda)(q+1)}{p+1} a^{p+1}
\end{aligned}
$$

where $\lambda=\frac{2(p-q)}{(q+1)(p-1)}$. Then

$$
\begin{aligned}
C \geq & E\left(u_{n}\right) \\
\geq & \frac{1}{2} \int_{\mathbb{R}^{N}}\left[\left|\nabla u_{n}\right|^{2}+V(x) u_{n}^{2}\right] \mathrm{d} x \\
& -\frac{\beta}{q+1}\left(\frac{\lambda(q+1)}{2} \int_{\mathbb{R}^{N}} V(x)\left|u_{n}\right|^{2} \mathrm{~d} x+\frac{(1-\lambda)(q+1)}{p+1} a^{p+1}\right) \\
\geq & \left(\frac{1}{2}-\frac{\beta(p-q)}{(q+1)(p-1)}\right)\left(\int_{\mathbb{R}^{N}}\left[\left|\nabla u_{n}\right|^{2}+V(x)\left|u_{n}\right|^{2}\right] \mathrm{d} x\right)-\frac{\beta(q-1)}{(q+1)(p-1)} a^{p+1}
\end{aligned}
$$

Because of $\beta<\frac{N-2}{2 N}, \frac{1}{2}-\frac{\beta(p-q)}{(q+1)(p-1)}>0$. It implies that $u_{n}(x)$ is bounded in $H_{V}^{1}\left(\mathbb{R}^{N}\right)$. By the compact embedding result from $H_{V}^{1}\left(\mathbb{R}^{N}\right)$ into $L^{r}\left(\mathbb{R}^{N}\right)$ for $2 \leq r<2^{*}$. We may assume that $u_{n} \rightarrow u_{b}$ in $H_{V}^{1}\left(\mathbb{R}^{N}\right), u_{n} \rightarrow u_{b}$ in $L^{r}\left(\mathbb{R}^{N}\right)$ for $2 \leq r<2^{*}$ and $u_{n}(x) \rightarrow u_{b}(x)$ a.e $x \in \mathbb{R}^{N}$. Hence $u_{b} \in M_{b}$, since $u_{n} \geq 0, u_{b} \geq 0$ and $u_{b} \neq 0$.

Using the same argument as the process of the proof of Lemma 2.1 in [20] and noting that $u_{n} \rightarrow u_{b}$ in $L^{q+1}\left(\mathbb{R}^{N}\right)$. We have

$$
\begin{aligned}
m_{b} & =\lim _{n \rightarrow \infty} E\left(u_{n}\right) \\
& \geq \liminf _{n \rightarrow \infty}\left\{\frac{1}{2} \int_{\mathbb{R}^{N}}\left[g^{2}\left(u_{n}\right)\left|\nabla u_{n}\right|^{2}+V(x) u_{n}^{2}\right] \mathrm{d} x-\frac{\beta}{q+1} \int_{\mathbb{R}^{N}}\left|u_{n}\right|^{q+1} \mathrm{~d} x\right\} \\
& \geq E\left(u_{b}\right)
\end{aligned}
$$

Hence $m_{b}$ is achieved at $u_{b}$ and 


$$
v_{b}=G\left(u_{b}\right) \in W_{b}, \quad F\left(v_{b}\right)=E\left(G^{-1}\left(v_{b}\right)\right)=E\left(u_{b}\right)=\omega_{b}
$$

and the property of $g$ implies $v_{b} \geq 0$ and $v_{b} \neq 0$.

Step 2: Set $h_{p}=\frac{1}{2 p} \int_{\mathbb{R}^{2 N}} \frac{\left|G^{-1}(v(x))\right|^{p}\left|G^{-1}(v(y))\right|^{p}}{|x-y|^{\mu}} \mathrm{d} x \mathrm{~d} y$ for $\frac{2 N-\mu}{N}<p<\frac{2 N-\mu}{N-2}$. Then $h_{p}(v) \in C^{1}\left(H_{V}^{1}\left(\mathbb{R}^{N}\right), \mathbb{R}\right)$. Actually, for any $\varphi \in H_{V}^{1}\left(\mathbb{R}^{N}\right)$, by Sobolev inequality and Hölder inequality, we get

$$
\begin{aligned}
\left|\left\langle h_{p}^{\prime}(v), \varphi\right\rangle\right| & =\left|\int_{\mathbb{R}^{N}} \frac{\left[|x|^{-\mu} *\left|G^{-1}(v)\right|^{p}\right]\left|G^{-1}(v(x))\right|^{r-2} G^{-1}(v(x))}{g\left(G^{-1}(v(x))\right)} \varphi\right| \mathrm{d} x \\
& \leq \int_{\mathbb{R}^{N}} \int_{\mathbb{R}^{N}} \frac{\left|G^{-1}(v(y))\right|^{p}\left|G^{-1}(v(x))\right|^{p-1}}{|x-y|^{\mu}}|\varphi| \mathrm{d} y \mathrm{~d} x \\
& \leq C\left\|G^{-1}(v(y))\right\|_{L^{p r}\left(\mathbb{R}^{N}\right)}^{p}\left\|G^{-1}(v(x))\right\|_{L^{p r}\left(\mathbb{R}^{N}\right)}^{p-1}\|\varphi\|_{L^{p r}\left(\mathbb{R}^{N}\right)} \\
& \leq C\|\varphi\|_{L^{p r}\left(\mathbb{R}^{N}\right)} \\
& \leq C\|\varphi\|_{H_{V}^{1}\left(\mathbb{R}^{N}\right)}
\end{aligned}
$$

where $r=\frac{2 N}{2 N-\mu}$ and $2 \leq p r<2^{*}$, so $h_{p}^{\prime}(v) \in\left(H_{V}^{1}\left(\mathbb{R}^{N}\right)\right)^{*}$.

Let $v_{n} \rightarrow v$ in $H_{V}^{1}\left(\mathbb{R}^{N}\right)$. Up to a subsequence, we can assume $v_{n} \rightarrow v$ a.e. in $\mathbb{R}$ and $v_{n} \rightarrow v$ in $L^{r}\left(\mathbb{R}^{N}\right)$ for $2 \leq r<2^{*}$. Hence

$$
\begin{aligned}
& \left|\left\langle h_{p}^{\prime}\left(v_{n}\right)-h_{p}^{\prime}(v), \varphi\right\rangle\right| \\
& =\mid \int_{\mathbb{R}^{N}} \frac{\left[|x|^{-\mu} *\left|G^{-1}\left(v_{n}\right)\right|^{p}\right]\left|G^{-1}\left(v_{n}\right)\right|^{p-2} G^{-1}\left(v_{n}\right)}{g\left(G^{-1}\left(v_{n}\right)\right)} \\
& -\frac{\left[|x|^{-\mu} *\left|G^{-1}(v)\right|^{p}\right]\left|G^{-1}(v)\right|^{p-2} G^{-1}(v)}{g\left(G^{-1}(v)\right)}|\varphi \mathrm{d} x| \\
& \leq\left|\int_{\mathbb{R}^{N}} \frac{\left[|x|^{-\mu} *\left(\left|G^{-1}\left(v_{n}\right)\right|^{p}-\left|G^{-1}(v)\right|^{p}\right)\right]\left|G^{-1}\left(v_{n}\right)\right|^{p-2} G^{-1}(v)}{g\left(G^{-1}\left(v_{n}\right)\right)} \varphi\right| \\
& \left.+\left|\int_{\mathbb{R}^{N}}\left[|x|^{-\mu} *\left|G^{-1}(v)\right|^{p}\right]\right| \frac{\left|G^{-1}\left(v_{n}\right)\right|^{p-1} G^{-1}\left(v_{n}\right)}{g\left(G^{-1}\left(v_{n}\right)\right)}-\frac{\left|G^{-1}(v)\right|^{p-1} G^{-1}(v)}{g\left(G^{-1}(v)\right)}\right) \varphi \mathrm{d} x \mid \\
& \leq C\left|\left\|\left.G^{-1}\left(v_{n}\right)\right|^{p}-\left|G^{-1}(v)\right|^{p}\right\|\right. \\
&
\end{aligned}
$$


Since $v_{n} \rightarrow v$ in $H_{V}^{1}\left(\mathbb{R}^{N}\right)$, we get $\left\|v_{n}\right\|_{H_{V}^{1}\left(\mathbb{R}^{N}\right)} \leq C, 2 \leq p r<2^{*}$ and by definition $g$, then

$$
\begin{aligned}
& \left\|\left|G^{-1}\left(v_{n}\right)\right|^{p}-\left|G^{-1}(v)\right|^{p}\right\|_{L^{r}\left(\mathbb{R}^{N}\right)}\left\|G^{-1}\left(v_{n}\right)\right\|_{L^{p r}\left(\mathbb{R}^{N}\right)}^{p-1}\|\varphi\|_{L^{p r}\left(\mathbb{R}^{N}\right)} \\
& \leq C\left(\left\|v_{n}\right\|_{L^{p r}\left(\mathbb{R}^{N}\right)}^{p}+\|v\|_{L^{p r}\left(\mathbb{R}^{N}\right)}^{p}\right),
\end{aligned}
$$

then by Lemma 3.4 [22] and assumption, we get

$$
\left\|\left|G^{-1}\left(v_{n}\right)\right|^{p}-\left|G^{-1}(v)\right|^{p}\right\|_{L^{r}\left(\mathbb{R}^{N}\right)}\left\|G^{-1}\left(v_{n}\right)\right\|_{L^{p r}\left(\mathbb{R}^{N}\right)}^{p-1}\|\varphi\|_{L^{p r}\left(\mathbb{R}^{N}\right)} \rightarrow 0, \quad n \rightarrow+\infty .
$$

Similarly,

$\left\|G^{-1}(v)\right\|_{L^{p r}\left(\mathbb{R}^{N}\right)}^{p}\left\|\left(\frac{\left|G^{-1}\left(v_{n}\right)\right|^{p-1} G^{-1}\left(v_{n}\right)}{g\left(G^{-1}\left(v_{n}\right)\right)}-\frac{\left|G^{-1}(v)\right|^{p-1} G^{-1}(v)}{g\left(G^{-1}(v)\right)}\right) \varphi\right\|_{L^{r}\left(\mathbb{R}^{N}\right)} \rightarrow 0, n \rightarrow+\infty$.

Hence, follows that $h_{p}(v) \in C^{1}\left(H_{V}^{1}\left(\mathbb{R}^{N}\right), \mathbb{R}\right)$ for $\frac{2 N-\mu}{N}<p<\frac{2 N-\mu}{N-2}$.

Step 3: For any $b \geq 0$, there exists $\lambda(b) \in \mathbb{R}$ such that $0<u_{b}=G^{-1}\left(v_{b}\right) \in M_{b}$ is a weak solution of Equation (1.1) with $\lambda=\lambda(b)$. In fact, by Lemma 2.4,

$$
\langle F(v), \varphi\rangle=\int_{\mathbb{R}^{N}} \nabla v \nabla \varphi+\int_{\mathbb{R}^{N}} \frac{V(x) G^{-1}(v)}{g\left(G^{-1}(v)\right)} \varphi \mathrm{d} x-\beta \int_{\mathbb{R}^{N}} \frac{\left|G^{-1}(v)\right|^{q-1} G^{-1}(v)}{g\left(G^{-1}(v)\right)} \varphi \mathrm{d} x
$$

and $F(v) \in\left(H_{V}^{1}\left(\mathbb{R}^{N}\right)\right)^{*}$ for all $v \in H_{V}^{1}\left(\mathbb{R}^{N}\right)$. Since $h_{p}^{\prime}(v) \in C\left(H_{V}^{1}\left(\mathbb{R}^{N}\right), \mathbb{R}\right)$ and $v_{b} \in W_{b}$, the implicit function theorem implies that for all $v \in \mathcal{N}\left(h_{p}^{\prime}\left(v_{b}\right)\right)$ (the null space of $h_{p}^{\prime}\left(v_{b}\right)$ ), there exist a $C^{1}$-map $g:[0,1] \rightarrow W_{b}$ such that $f(0)=v_{a}$ and $g^{\prime}(0)=v$. Now, we prove $\left\langle F\left(v_{b}\right), v\right\rangle=0$ for all $v \in \mathcal{N}\left(h_{p}^{\prime}\left(v_{b}\right)\right)$. Indeed, for every $t>0, f(t)=v_{b}+v+o(t) \in W_{b}$, where $\frac{o(t)}{t} \rightarrow 0$ as $t \rightarrow 0$. Let

$$
\Phi(r)=F\left(v_{b}+r(t v+o(t))\right)
$$

By Lemma 2.4

$$
\begin{aligned}
\Phi^{\prime}(r) & =\lim _{\delta \rightarrow 0} \frac{\Phi(r+\delta)-\Phi(r)}{\delta} \\
& =\lim _{\delta \rightarrow 0} \frac{F\left(v_{a}+(r+\delta)(t v+o(t))\right)-F\left(v_{a}+r(t v+o(t))\right)}{\delta} \\
& =\left\langle F^{\prime}\left(v_{b}+r(t v+o(t))\right), t v+o(t)\right\rangle .
\end{aligned}
$$

Hence there exist an $\theta \in(0,1)$ such that

$$
\begin{aligned}
& F\left(v_{b}+t v+o(t)\right)-F\left(v_{b}\right)=\left\langle F^{\prime}\left(v_{b}+\theta(t v+o(t))\right), t v+o(t)\right\rangle \\
& =t\left\langle F^{\prime}\left(v_{b}+\theta(t v+o(t))\right), v\right\rangle+t\left\langle F^{\prime}\left(v_{a}+\theta(t v+o(t))\right), \frac{o(t)}{t}\right\rangle .
\end{aligned}
$$

Take limit $t \rightarrow 0$, by Lemma 2.4, one has $F^{\prime}\left(v_{b}+\theta(t v+o(t))\right) \rightarrow F^{\prime}\left(v_{b}\right)$ weakly. It follows that $\left\langle F^{\prime}\left(v_{b}+\theta(t v+o(t))\right), v\right\rangle \rightarrow\left\langle F^{\prime}\left(v_{a}\right), v\right\rangle$ and 
$\left\{F^{\prime}\left(v_{b}+\theta(t v+o(t))\right)\right\}$ is bounded. Since $\frac{o(t)}{t} \rightarrow 0$ as $t \rightarrow 0$, we have $\left\langle F_{b}^{\prime}\left(v_{b}+\theta(t v+o(t))\right), \frac{o(t)}{t}\right\rangle \rightarrow 0$.

Since $F\left(v_{b}\right)=\omega_{b}$, one has

$$
\begin{aligned}
& 0 \leq F\left(v_{b}+t v+o(t)\right)-F\left(v_{b}\right) \\
& =t\left\langle F^{\prime}\left(v_{b}+\theta(t v+o(t))\right), v\right\rangle+t\left\langle F^{\prime}\left(v_{b}+\theta(t v+o(t))\right), \frac{o(t)}{t}\right\rangle .
\end{aligned}
$$

Hence

$$
0 \leq\left\langle F_{b}^{\prime}\left(v_{a}+\theta(t v+o(t))\right), v\right\rangle+\left\langle F^{\prime}\left(v_{a}+\theta(t v+o(t))\right), \frac{o(t)}{t}\right\rangle .
$$

Take limit $t \rightarrow 0$, we get $\left\langle F^{\prime}\left(v_{b}\right), v\right\rangle \geq 0$. By arbitrariness of $v$, one has $\left\langle F^{\prime}\left(v_{b}\right),-v\right\rangle \geq 0$. It follows that $\left\langle F^{\prime}\left(v_{b}\right), v\right\rangle=0$, for every $v \in \mathcal{N}\left(h_{p}^{\prime}\left(v_{b}\right)\right)$. Set $v^{\prime} \in H_{V}^{1}\left(\mathbb{R}^{N}\right)$ be such that $\left\langle h_{p}^{\prime}\left(v_{a}\right), v^{\prime}\right\rangle=1$, for every $\varphi \in H_{V}^{1}\left(\mathbb{R}^{N}\right)$, let

$$
\psi=\varphi-\left\langle h_{p}^{\prime}\left(v_{b}\right), \varphi\right\rangle v^{\prime} .
$$

Then $\psi \in \mathcal{N}\left(h_{p}^{\prime}\left(v_{b}\right)\right)$. It means $\left\langle F^{\prime}\left(v_{b}\right), \psi\right\rangle=0$, i.e.

$$
\left\langle F^{\prime}\left(v_{b}\right), \varphi\right\rangle=\left\langle F^{\prime}\left(v_{b}\right), v^{\prime}\right\rangle\left\langle h_{p}^{\prime}\left(v_{a}\right), \varphi\right\rangle .
$$

Put $\lambda=\lambda(b)=\left\langle F^{\prime}\left(v_{b}\right), v^{\prime}\right\rangle$, we have

$$
\left\langle F^{\prime}\left(v_{b}\right), \varphi\right\rangle=\lambda\left\langle h_{p}^{\prime}\left(v_{b}\right), \varphi\right\rangle,
$$

namely,

$$
\begin{aligned}
& \int_{\mathbb{R}^{N}} \nabla v_{b} \nabla \varphi \mathrm{d} x+\int_{\mathbb{R}^{N}} \frac{V(x) G^{-1}\left(v_{b}\right)}{g\left(G^{-1}\left(v_{b}\right)\right)} \varphi \mathrm{d} x \\
& =\lambda \int_{\mathbb{R}^{N}}\left[|x|^{-\mu} *\left|G^{-1}\left(v_{b}\right)\right|^{p}\right] \frac{\left|G^{-1}\left(v_{b}\right)\right|^{p-2} G^{-1}\left(v_{b}\right)}{g\left(G^{-1}\left(v_{b}\right)\right)} \varphi \mathrm{d} x \\
& \quad+\beta \int_{\mathbb{R}^{N}} \frac{\left|G^{-1}\left(v_{b}\right)\right|^{q-1} G^{-1}\left(v_{b}\right)}{g\left(G^{-1}\left(v_{b}\right)\right)} \varphi \mathrm{d} x .
\end{aligned}
$$

It implies that $u_{b}=G^{-1}\left(v_{b}\right) \in M_{b}$ is a weak solution of Equation (1.1). Moreover, the maximum principle implies $u_{b}>0$.

\section{Acknowledgements}

This work is supported by the Natural Science Foundation of China (11961081).

\section{Conflicts of Interest}

The authors declare no conflicts of interest regarding the publication of this paper.

\section{References}

[1] Pekar, S. (1945) Untersuchungen über die Elektronentheorie der Kristale. Akademie 
Verlag, Berlin.

[2] Lieb, E.H. (1977) Existence and Uniqueness of the Minimizing Solution of Choquard's Nonlinear Equation. Studies in Applied Mathematics, 57, 93-105. https://doi.org/10.1002/sapm197757293

[3] Penrose, R. (1996) On Gravity's Role in Quantum State Reductions. General Relativity and Gravitation, 28, 581-600. https://doi.org/10.1007/BF02105068

[4] Lions, P.L. (1980) The Choquard Equation and Related Questions. Nonlinear Analysis: Theory, Methods \& Applications, 4, 1063-1072. https://doi.org/10.1016/0362-546X(80)90016-4

[5] Ma, L. and Zhao, L. (2010) Classification of Positive Solitary Solutions of the Nonlinear Choquard Equation. Archive for Rational Mechanics and Analysis, 195, 455-467. https://doi.org/10.1007/s00205-008-0208-3

[6] Cingolani, S., Clapp, M. and Secchi, S. (2012) Multiple Solutions to a Magnetic Nonlinear Choquard Equation. Zeitschrift für Angewandte Mathematik und Physik, 63, 233-248. https://doi.org/10.1007/s00033-011-0166-8

[7] Moroz, V. and Van Schaftingen, J. (2013) Groundstates of Nonlinear Choquard Equations: Existence, Qualitative Properties and Decay Asymptotics. Journal of Functional Analysis, 265, 153-184. https://doi.org/10.1016/j.jfa.2013.04.007

[8] Moroz, V. and Van Schaftingen, J. (2015) Existence of Groundstates for a Class of Nonlinear Choquard Equations. Transactions of the American Mathematical Society, 367, 6557-6579. https://doi.org/10.1090/S0002-9947-2014-06289-2

[9] Clapp, M. and Salazar, D. (2013) Positive and Sign Changing Solutions to a Nonlinear Choquard Equation. Journal of Mathematical Analysis and Applications, 407, 1-15. https://doi.org/10.1016/j.jmaa.2013.04.081

[10] Ghimenti, M. and Van Schaftingen, J. (2016) Nodal Solutions for the Choquard Equation. Journal of Functional Analysis, 271, 107-135.

https://doi.org/10.1016/j.jfa.2016.04.019

[11] Wei, J.C. and Winter, M. (2009) Strongly Interacting Bumps for the SchrödingNewton Equation. Journal of Mathematical Physics, 50, Article ID: 012905. https://doi.org/10.1063/1.3060169

[12] Moroz, V. and Van Schaftingen, J. (2015) Semi-Classical States for the Choquard Equation. Calculus of Variations and Partial Differential Equations, 52, 199-235. https://doi.org/10.1007/s00526-014-0709-x

[13] Moroz, V. and Van Schaftingen, J. (2015) Groundstates of Nonlinear Choquard Equations: Hardy-Littlewood-Sobolev Critical Exponent. Communications in Contemporary Mathematics, 17, Article ID: 1550005. https://doi.org/10.1142/S0219199715500054

[14] Cassani, D. and Zhang, J. (2018) Choquard-Type Equations with Hardy-LittlewoodSobolev Upper-Critical Growth. Advances in Nonlinear Analysis, 8, 1184-1212. https://doi.org/10.1515/anona-2018-0019

[15] Li, Q.Q., Teng, K. and Zhang, J. (2020) Ground State Solutions for Fractional Choquard Equations Involving Upper Critical Exponent. Nonlinear Analysis, 197, Article ID: 111846. https://doi.org/10.1016/j.na.2020.111846

[16] Seok, J. (2018) Limit Profiles and Uniqueness of Ground States to the Nonlinear Choquard Equations. Advances in Nonlinear Analysis, 8, 1083-1098. https://doi.org/10.1515/anona-2017-0182

[17] Li, Q.Q., Teng, K.M., Zhang, J. and Nie, J.J. (2020) An Existence Result for a Generalized Quasilinear Schrödinger Equation with Nonlocal Term. Journal of Functional 
Spaces, 2020, Article ID: 6430104. https://doi.org/10.1155/2020/6430104

[18] Chen, S.X. (2013) Existence of Positive Solutions for a Class of Quasilinear Schrödinger Equations on $\mathbb{R}^{N}$. Journal of Mathematical Analysis and Applications, 405, 595-607. https://doi.org/10.1016/j.jmaa.2013.04.031

[19] Li, Q.Q. and Wu, X. (2016) Multiple Solutions for Genneralized Quasilinear Schrödinger Equations. Mathematical Methods in the Applied Science, 40, 1359-1366. https://doi.org/10.1002/mma.4050

[20] Shen, Y.T. and Wang, Y.J. (2013) Soliton Solutions for Genneralized Quasilinear Schrödinger Equations. Nonlinear Analysis, 80, 194-201.

https://doi.org/10.1016/j.na.2012.10.005

[21] Liu, J.Q., Wang, Y.Q. and Wang, Z.Q. (2003) Soliton Solutions for Quasilinear Schrödinger Equations: II. Journal of Differential Equations, 187, 473-493. https://doi.org/10.1016/S0022-0396(02)00064-5

[22] Chen, S.X. and Wu, X. (2019) Existence of Positive Solutions for a Class of Quasilinear Schrödinger Equations of Choquard Type. Journal of Mathematical Analysis and Applications, 475, 1754-1777. https://doi.org/10.1016/j.jmaa.2019.03.051

[23] Liu, J., Wang, Y. and Wang, Z.Q. (2003) Soliton Solutions for Quasilinear Schrödinger Equations: I. Proceedings of the American Mathematical Society, 131, 441-448. https://doi.org/10.1090/S0002-9939-02-06783-7 